\title{
Statistics of Random Permutations and the Cryptanalysis Of Periodic Block Ciphers
}

\author{
Nicolas T. Courtois, Gregory V. Bard, and Shaun V. Ault \\ Communicated by $\mathrm{xxx}$
}

\begin{abstract}
A block cipher is intended to be computationally indistinguishable from a random permutation of appropriate domain and range. But what are the properties of a random permutation? By the aid of exponential and ordinary generating functions, we derive a series of collolaries of interest to the cryptographic community. These follow from the Strong Cycle Structure Theorem of permutations, and are useful in rendering rigorous two attacks on Keeloq, a block cipher in wide-spread use. These attacks formerly had heuristic approximations of their probability of success.

Moreover, we delineate an attack against the (roughly) millionth-fold iteration of a random permutation. In particular, we create a distinguishing attack, whereby the iteration of a cipher a number of times equal to a particularly chosen highly-composite number is breakable, but merely one fewer round is considerably more secure. We then extend this to a key-recovery attack in a "Triple-DES" style construction, but using AES-256 and iterating the middle cipher (roughly) a million-fold.

It is hoped that these results will showcase the utility of exponential and ordinary generating functions and will encourage their use in cryptanalytic research.
\end{abstract}

Keywords. Generating Functions, EGF, OGF, Random Permutations, Cycle Structure, Cryptanalysis, Iterations of Permutations, Analytic Combinatorics, Keeloq.

AMS classification. 05A15, 94A60, 20B35, $11 \mathrm{~T} 71$.

\section{Introduction}

The technique of using a function of a variable to count objects of various sizes, using the properties of multiplication and addition of series as an aid, is accredited to PierreSimon Laplace [12]. Here, we will use this family of techniques, now called "analytic combinatorics" to count permutations of particular types. An ordinary generating series associated with a set of objects assigns as the coefficient of the $z^{i}$ th term, the number of objects of size $i$. An exponential generating series is merely this, with each term divided by $i$ !. In particular, this can be used to describe permutations drawn at random from $S_{n}$, which is the topic of this paper.

The cipher Keeloq, can be written as the eighth iterate of a permutation followed by one more permutation [3, Ch. 2]. This eighth power naturally affects the cycle structure; for example, we will prove that the fixed points of the eighth power are those of order $\{1,2,4,8\}$ under the original. There are many other properties of these repeated permutations that follow from the factorization of the number of iterations, and we will show cryptanalytic consequences.

In the remainder of this section we will introduce analytic combinatorics through exponential and ordinary generating functions. In Section 2 we prove a theorem on 
the cycle structures of random permutations, and in Section 3 we present a number of corollaries. We imagine that most if not all of these are already known in some form, but here we are compiling them all in one place, with a view to determining when a random permutation has a given property, rather than merely counting objects which is the usual use of the techniques of this subject. The proofs are our own. In Section 4, we apply these techniques to Keeloq, and describe two quite feasible attacks, but also their exact success probabilities. These attacks have been previously described as requiring the entire code-book of the cipher (all plaintext-ciphertext pairs under the current key) but here we let $\eta$ represent the fraction of the code-book available, and show how $\eta$ affects the success probability. In Section 5, we present an unusual example, where a very highly iterated cipher appears to be secure, but adding one iteration opens up a feasible and effective distinguishing attack. We conclude in Section 6.

\subsection{Background}

A combinatorial class $\mathcal{C}$ is a set of objects $C$ together with a function $\ell_{C}: C \rightarrow \mathbb{Z}^{\geq 0}$, which asssigns to each element a non-negative integer "size". For example, if $P$ is the set of permutation groups $S_{n}$ for all positive integers $\mathrm{n}$, then we may use the size function $\ell_{P}(\pi)=n$, for any $\pi \in S_{n}$, to make $\mathcal{P}$ into a combinatorial class.

Let $C_{i}$ be the cardinality of the set of elements of $C$ with size $i$. Thus in our example, $P_{i}=i$ ! for $i \geq 0$. It will be useful to represent $C_{i}$ by either an exponential or an ordinary generating function (OGF or EGF). First, a brief discussion of generating functions is in order.

Given a set of constants indexed by $\mathbb{Z}^{\geq 0}$, say $c_{0}, c_{1}, c_{2}, \ldots$, the ordinary generating function (or OGF) is defined as the formal power series:

$$
c(z) \stackrel{\text { def }}{=} \sum_{i=0}^{\infty} c_{i} z^{i}=c_{0}+c_{1} z+c_{2} z^{2}+c_{3} z^{3}+\cdots .
$$

The EGF is defined as the formal power series:

$$
c_{e}(z) \stackrel{\text { def }}{=} \sum_{i=0}^{\infty} \frac{c_{i}}{i !} z^{i}=c_{0}+\frac{c_{1}}{1 !} z+\frac{c_{2}}{2 !} z^{2}+\frac{c_{3}}{3 !} z^{3}+\cdots .
$$

For our example combinatorial class, $\mathcal{P}$, its OGF is $\mathcal{P}(z)=z+2 z^{2}+6 z^{3}+24 z^{4}+$ $120 z^{5}+\cdots$, and its EGF is $\mathcal{P}_{e}(z)=z+z^{2}+z^{3}+z^{4}+z^{5}+\cdots$. The series $1+$ $z+z^{2}+z^{3}+z^{4}+z^{5}+\cdots$ represents the OGF of the non-negative integers, $\mathbb{Z}^{\geq 0}$ with "size" function being the identity: $\ell(n)=n$.

In combinatorial arguments, OGFs and EGFs abound [12] [15] and are especially useful in counting partitions of sets. For example, let $A_{1}, A_{2}, \ldots, A_{k}$ be sets of whole numbers. The number of all distinct ways that $n$ identical objects can be placed into $k$ containers, where container $j$ must have some number of objects that occurs in the set $A_{j}$ will be the coefficient of $z^{n}$ in the OGF:

$$
\left(\sum_{i \in A_{1}} z^{i}\right)\left(\sum_{i \in A_{2}} z^{i}\right) \cdots\left(\sum_{i \in A_{k}} z^{i}\right)
$$


a function that we will use in the proof of Lemma 2.3. Notice that the $j^{\text {th }}$ factor is the OGF that represents the set $A_{j}$. There is a similar interpretation for EGFs and products of EGFs, in terms of probability rather than strict counting. See Section 3.2 or Theorem 3.8 as an example.

A less trivial example of a combinatorial class is the class $\mathcal{O}$ of $n$-cycles of $S_{n}$, for all $n>0$, with size function $\ell(\pi)=n$ if $\pi \in S_{n}$. In other words, size $n$ members of $\mathcal{O}$ comprise the subset of permutations of $S_{n}$ where the permutation has exactly one orbit. For any $n>0$ there are $n ! / n$ or $(n-1) !$ of these. Thus the OGF is $z+z^{2}+2 z^{3}+$ $6 z^{4}+24 z^{5}+120 z^{6}+\cdots$, and the EGF is $z+z^{2} / 2+z^{3} / 3+z^{4} / 4+z^{5} / 5+z^{6} / 6+\cdots$. Thus the probability that a random permutation from $S_{n}$ has only one cycle is given by the coefficients of the $z^{n}$ terms in the EGF. Namely, $(n-1) ! / n !=1 / n$.

Often, the formal power series defining OGFs or EGFs converge to functions (in some neighborhood of 0 ). For example, the OGF for $\mathbb{Z}^{\geq 0}$ converges to $1 /(1-z)$, and its EGF converges to $e^{z}$. The EGF for the combinatorial class $\mathcal{O}$ above also converges:

$$
z+\frac{z^{2}}{2}+\frac{z^{3}}{3}+\frac{z^{4}}{4}+\frac{z^{5}}{5}+\frac{z^{6}}{6}+\cdots=\log \left(\frac{1}{1-z}\right),
$$

as can be verified by term-by-term integration of the power series for $\frac{1}{1-z}$. The existence of such functions will facilitate multiplications and compositions.

\subsection{Notation}

The somewhat unusual notation of $\exp (C)$ where $C$ is a series, means precisely substituting the entire series $C$ for $z$ into the Taylor expansion for $e^{z}=\sum_{i \geq 0} z^{i} / i$ !, similar to matrix exponentiation.

It is well-known that any permutation may be written uniquely as a product of disjoint cycles, up to reordering of the cycles and cyclic reordering within each cycle; indeed, for any given permutation $\pi$ consisting of $k$ disjoint cycles, having cycle lengths $c_{1}, c_{2}, c_{3}, \ldots, c_{k}$, there are exactly $k ! c_{1} c_{2} c_{3} \cdots c_{k}$ ways to reorder to obtain an equivalent expression for $\pi$. Any counts we make of symmetric group elements must take this fact into account. Note, we use the convention that if $\pi$ has a fixed-point, $a$, then the 1-cycle $(a)$ is part of the expression for $\pi$ as disjoint cycles. In particular, the identity of $S_{n}$ is written (1)(2)(3) $\cdots(n)$. We use the term cycle-count for the number of disjoint cycles (including all 1-cycles) in the expression of a permutation. It shall be convenient to include in our analysis the unique permutation of no letters, which has by convention cycle-count 0 . We may view this element as the sole member of $S_{0}$.

\section{Strong and Weak Cycle Structure Theorems}

Let $A$ be a subset of the positive integers. We consider the class of permutations that consist entirely of disjoint cycles of lengths in $A$, and denote this by $\mathcal{P}^{\left(A, \mathbb{Z}^{\geq 0}\right)}$. Furthermore, if $B \subseteq \mathbb{Z}^{\geq 0}$, we may consider the subclass $\mathcal{P}^{(A, B)} \subseteq \mathcal{P}^{\left(A, \mathbb{Z}^{\geq 0}\right)}$ consisting of only those permutations whose cycle count is found in $B$. That is, any permutation of cycle count not in $B$, or containing a cycle length not in $A$, are prohibited. 
The following theorems were first proven (presumably) long ago but can be found in [12] and also [15], and it is commonly noted that the technique in general was used by Laplace in the late 18th century. The nomenclature is however, ours.

Theorem 2.1. The Strong Cycle Structure Theorem:

The combinatorial class $\mathcal{P}^{(A, B)}$ has associated EGF, $\mathcal{P}_{e}^{(A, B)}(z)=\beta(\alpha(z))$, where $\beta(z)$ is the EGF associated to $B$ and $\alpha(z)=\sum_{i \in A} \frac{z^{i}}{i}$.

However, we only need a weaker form in all but one case in this paper:

Theorem 2.2. The Weak Cycle Structure Theorem:

The combinatorial class $\mathcal{P}^{\left(A, \mathbb{Z}^{\geq 0}\right)}$ has associated $E G F, \mathcal{P}_{e}^{\left(A, \mathbb{Z}^{\geq 0}\right)}(z)=\exp (\alpha(z))$, where $\alpha(z)$ is as above: $\alpha(z)=\sum_{i \in A} \frac{z^{i}}{i}$

This is clearly a special case of the Strong Cycle Structure Theorem with $\beta(z)=$ $1+z+z^{2} / 2 !+z^{3} / 3 !+z^{4} / 4 !+\cdots=e^{z}$ (the EGF of $\mathbb{Z}^{\geq 0}$ ). Interestingly, if $A=\mathbb{Z}^{+}$, then $\alpha(z)=z+z^{2} / 2+z^{3} / 3+z^{4} / 4+z^{5} / 5+\cdots=\log \left(\frac{1}{1-z}\right)$, which provides a verification of the theorem in this special case:

$$
\exp \left(\log \left(\frac{1}{1-z}\right)\right)=\frac{1}{1-z}=1+z+z^{2}+z^{3}+z^{4}+\cdots,
$$

which is the EGF for the combinatorial class $\mathcal{P}$ of all permutations (together with the unique permutation on 0 letters), as expected.

Since the proof of the strong version is not fundamentally more difficult than the weak version, we shall provide a proof of Theorem 2.1. While this has been proven already in [12], we feel that a more expository proof is appropriate in this context. First, a lemma which proves the case $B=\{k\}$.

Lemma 2.3. The combinatorial class $\mathcal{P}^{(A,\{k\})}$ has associated EGF,

$$
\mathcal{P}_{e}^{(A,\{k\})}(z)=\frac{1}{k !}\left(\sum_{i \in A} \frac{z^{i}}{i}\right)^{k} .
$$

Proof. Let $A \subseteq \mathbb{Z}^{+}$. For a given cycle-count, $k$, we must only include cycles of lengths found in $A$. Begin with an OGF. If $\pi \in S_{n}$ has $k$ cycles, then its cycle structure defines a partition of $n$ identical objects into $k$ containers, where each container cannot have any number of objects that does not occur as a member of $A$. The OGF that generates this is $\left(\sum_{i \in A} z^{i}\right)^{k}$, as stated in Section 1.1. Now, we must remember that those objects in the containers are not identical! Think of each cycle-structure as being a template onto which we attach the labels $1,2,3,4, \ldots, n$ in some order. A priori, this provides a factor of $n$ ! for each partition of $n$, and so the coefficient of $z^{n}$ in the above OGF should be multiplied by $n$ !. The best way to accomplish this is to 
simply consider our OGF as an EGF: In our OGF, if $C_{n}$ is the coeffiecient of $z^{n}$, then as EGF, $n ! C_{n}$ is the coefficient of $z^{n} / n$ !. Now, for each disjoint cycle of length $i$, there are $i$ ways of cyclically permuting the labels, each giving rise to an equivalent representaion of the same $i$-cycle. Thus, we have over-counted unless we divide each term $z^{i}$ by $i$. Finally, each rearrangement of the $k$ cycles among themselves gives rise to an equivalent expression for the permuation, so we must divide by $k$ !, and our EGF for permutations of cycle-count $k$ with cycle-lengths in $A$ now has the required form, $\mathcal{P}_{e}^{(A,\{k\})}(z)=\frac{1}{k !}\left(\sum_{i \in A} z^{i} / i\right)^{k}$.

The proof of Theorem 2.1 then follows easily:

Proof. Let $A \subseteq \mathbb{Z}^{+}, B \subseteq \mathbb{Z}^{\geq 0}$. Categorize all permutations in $\mathcal{P}$ by cycle-count. Only permutations with cycle-counts $k \in B$ will contribute to our total, so by Lemma 2.3,

$$
\mathcal{P}_{e}^{(A, B)}(z)=\sum_{k \in B} \mathcal{P}_{e}^{(A,\{k\})}(z)=\sum_{k \in B} \frac{1}{k !}\left(\sum_{i \in A} \frac{z^{i}}{i}\right)^{k}=\sum_{k \in B} \frac{\alpha(z)^{k}}{k !}=\beta(\alpha(z)),
$$

since $\sum_{k \in B} z^{k} / k$ ! is the EGF associated to $B$. The Weak Cycle Structure Theorem then follows as an immediate corollary.

\subsection{Probabilities}

In cryptography and other disciplines, we are often concerned with determining whether or not a random permutation has some given property $\phi$. We can calculate then the OGF of the combinatorial class $\mathcal{F}$ of permutations with that property, and divide term-wise with the same term from the OGF of $\mathcal{P}$, the combinatorial class of all permutations. But this is the same as the coefficients of the EGF of $\mathcal{F}$.

This works for any specific size, but first, it might be difficult to calculate, and second we might want to know the limit of this probability as the size goes to infinity.

Theorem 2.4. Let $\mathcal{F} \subset \mathcal{P}$ be the combinatorial class of permutations with property $\phi$. Suppose further $\mathcal{F}$ has EGF equal to $f(z)$. Then the limit (as $n$ goes to infinity) of the probability that a random permutation of size $n$ has property $\phi$ is given by

$$
p=\lim _{z \rightarrow 1^{-}}(1-z) f(z)
$$

provided that $(1-z) f(z)$ is continuous from the left at $z=1$.

Proof. Let the OGF of $\mathcal{F}$ be given by $A_{0}+A_{1} z+A_{2} z^{2}+A_{3} z^{3}+A_{4} z^{4}+A_{5} z^{5}+\cdots$. Consider the following function

$$
g_{n}(z)=\frac{A_{0}}{0 !}+\sum_{1 \leq i \leq n}\left(\frac{A_{i}}{i !}-\frac{A_{i-1}}{(i-1) !}\right) z^{i},
$$

which when evaluated at $z=1$, the sum telescopes,

$$
=\frac{A_{0}}{0 !}+\left(\frac{A_{1}}{1 !}-\frac{A_{0}}{0 !}\right)(1)+\left(\frac{A_{2}}{2 !}-\frac{A_{1}}{1 !}\right)(1)^{2}+\cdots+\left(\frac{A_{n}}{n !}-\frac{A_{n-1}}{(n-1) !}\right)(1)^{n}=\frac{A_{n}}{n !} \text {. }
$$


Thus $g_{n}(1)$ is the desired probability, for size $n$.

The limit $g(z)=\lim _{n \rightarrow \infty} g_{n}(z)=\frac{A_{0}}{0 !}+\sum_{i \geq 1}\left(\frac{A_{i}}{i !}-\frac{A_{i-1}}{(i-1) !}\right) z^{i}$ does not necessarily exist for all $z$, but when it does, we have

$$
\begin{aligned}
g(z)=\lim _{n \rightarrow \infty} g_{n}(z) & =\lim _{n \rightarrow \infty} \frac{A_{0}}{0 !}+\left(\sum_{i=1}^{n} \frac{A_{i}}{i !} z^{i}\right)-\left(\sum_{i=1}^{n} \frac{A_{i-1}}{(i-1) !} z^{i}\right) \\
& =\lim _{n \rightarrow \infty}\left(\sum_{i=0}^{n} \frac{A_{i}}{i !} z^{i}\right)-z\left(\sum_{j=0}^{n} \frac{A_{j}}{j !} z^{j}\right) \\
& =(1-z) \lim _{n \rightarrow \infty}\left(\sum_{i=0}^{n} \frac{A_{i}}{i !} z^{i}\right)=(1-z) f(z)
\end{aligned}
$$

Thus $p=\lim _{n \rightarrow \infty} g_{n}(1)=\lim _{n \rightarrow \infty} \lim _{z \rightarrow 1^{-}} g_{n}(z)=\lim _{z \rightarrow 1^{-}}(1-z) f(z)$.

Note, we implicitly assumed that $g(z)$ is continuous (from the left) near $z=1$ in order to reverse the order of the limits in the last step, but this will be the case in all of our examples.

\subsection{Expected Values}

While OGFs and EGFs are very useful for the study of a one-parameter family of constants, $A_{0}, A_{1}, A_{2}, A_{3}, \ldots$, we often wish to work with a two-parameter family, $\left\{A_{s, t}\right\}_{s, t>0}$. This is accomplished using double generating functions. The double OGF, $A(y, z)$ of a two-parameter family of constants, $\left\{A_{s, t}\right\}$ is defined to be the formal sum:

$$
A(y, z)=\sum_{s=0}^{\infty} \sum_{t=0}^{\infty} A_{s, t} y^{s} z^{t},
$$

and the EGF $A_{e}(y, z)$ is defined to be the formal sum:

$$
A_{e}(y, z)=\sum_{s=0}^{\infty} \sum_{t=0}^{\infty} \frac{A_{s, t}}{(s+t) !} y^{s} z^{t}
$$

For our purposes, we will be interested in a combinatorial class of permutations categorized not only by the order of the symmetric group $S_{n}$ in which the permutation lies, but also by the number of fixed points that the permutation possesses.

Theorem 2.5. Let $\mathcal{F} \subset \mathcal{P}$ be a combinatorial class of permutations with double $E G F$ $a(y, z)$, where the coefficient of $y^{s} z^{t} /(s+t)$ ! is the number of permutations $\pi$ with property $\phi_{s}$ such that $\pi \in S_{s+t}$. Then the limit (as $n=s+t$ goes to infinity) of the expected value of $s$ such that a random permutation of size $n$ satisfies $\phi_{s}$ is given by:

$$
\lim _{z \rightarrow 1^{-}}(1-z) a_{y}(z, z)
$$

provided $(1-z) a_{y}(z, z)$ is convergent and continuous from the left at $z=1$. 
Proof. Let $a(y, z)=\sum_{s>0} \sum_{t>0} y^{s} z^{t} A_{s, t} /(s+t)$ !. The coefficient of $y^{s} z^{t}$ is the probability that a random permutation of $S_{s+t}$ has property $\phi_{s}$, by construction. Consider the partial derivative with respect to $y$ :

$$
a_{y}(y, z)=\sum_{s \geq 0} \sum_{t \geq 0} \frac{s A_{s, t}}{(s+t) !} y^{s-1} z^{t} .
$$

The probabilities are now multiplied by the corresponding value of $s$. Now, letting $y=z$ produces:

$$
a_{y}(z, z)=\sum_{s \geq 0} \sum_{t \geq 0} \frac{s A_{s, t}}{(s+t) !} z^{s+t-1}=\sum_{n \geq 0}\left(\sum_{s+t=n} \frac{s A_{s, t}}{n !}\right) z^{n-1} .
$$

Thus, $a_{y}(z, z)$ is the OGF that computes the expected value of $s$ such that a random permutation of size $n$ satisfies $\phi_{s}$ (shifted by one degree). Using the same technique as in the proof of Thm 2.4, we find that

$$
\lim _{z \rightarrow 1^{-}}(1-z) a_{y}(z, z)=\lim _{n \rightarrow \infty}\left(\sum_{s+t=n} \frac{s A_{s, t}}{n !}\right) .
$$

\section{Corollaries}

Theorem 2.4 is exploited extensively in a paper by Marko R. Riedel dedicated to random permutation statistics, but in a different context (see [15]).

Corollary 3.1. The probability that a random permutation (in the limit as the size grows to infinity) does not contain cycles of length $k$ is given by $e^{-1 / k}$.

Proof. The set $A$ of allowable cycle lengths is $\mathbb{Z}^{+}-\{k\}$, and so has EGF given by artificially removing the term for $k$ from the EGF of $\mathcal{O}$ :

$$
z+\frac{z^{2}}{2}+\frac{z^{3}}{3}+\cdots+\frac{z^{k-1}}{k-1}+0+\frac{z^{k+1}}{k+1}+\frac{z^{k+2}}{k+2}+\cdots=\log \left(\frac{1}{1-z}\right)-\frac{z^{k}}{k},
$$

and thus by the Weak Cycle Structure Theorem, the combinatorial class in question has EGF equal to

$$
a(z)=\exp \left(\log \left(\frac{1}{1-z}\right)-\frac{z^{k}}{k}\right)=\frac{1}{1-z} e^{-z^{k} / k}
$$

Thus the probability of a random permutation (as the size tends toward infinity) not having any cycles of length $k$ is given by $\lim _{z \rightarrow 1^{-}}(1-z) a(z)=e^{-1 / k}$ 
Note: On the Precision of these estimations: This result means that $p \rightarrow e^{-\frac{1}{k}}$ when $N \rightarrow \infty$. What about when $N=2^{32}$ ? We can answer this question easily by observing that the Taylor expansion of the function $a(z)$ is the EGF and therefore gives all the exact values of $A_{n} / n$ !. For example when $k=4$ we computed the Taylor expansion of $g(z)$ at order 201, where each coefficient is a computed as a ratio of two large integers. This takes less than a second with the computer algebra software Maple [14]. The results are surprisingly precise: the difference between the $A_{200} / 200$ ! and the limit is less than $2^{-321}$. Thus convergence is very fast and even for very small permutations (on 200 elements).

Returning to the proving of corollaries, let us define $\mathcal{P}^{\bar{A}}=\mathcal{P}^{\left(\mathbb{Z}^{+}-A, \mathbb{Z}^{\geq 0}\right)}$ and find its EGF.

Lemma 3.2. The EGF of $\mathcal{P}^{\bar{A}}$ is given by $\exp (f(z))$, where

$$
f(z)=\sum_{i \notin A} z^{i} / i=\log \left(\frac{1}{1-z}\right)-\sum_{i \in A} z^{i} / i
$$

Proof. Because $\mathcal{P}^{\bar{A}}=\mathcal{P}^{\left(\mathbb{Z}^{+}-A, \mathbb{Z}^{\geq 0}\right)}$ we can use the Weak Cycle Structure Theorem. The EGF of the combinatorial class of cycles with size from the set $\mathbb{Z}^{+}-A$ is given by that of $\mathcal{O}$ (the class of all cycles) with the "forbidden lengths" artificially set to zero, namely

$$
\sum_{i \in\left(\mathbb{Z}^{+}-A\right)} z^{i} / i=\sum_{0<i \notin A} z^{i} / i=\log \left(\frac{1}{1-z}\right)-\sum_{i \in A} z^{i} / i
$$

The correct answer follows.

Corollary 3.3. Let $A$ be a subset of the positive integers. The probability that a random permutation (in the limit as the size grows to infinity) does not contain cycles of length in $A$ is:

$$
\prod_{i \in A} e^{-1 / i}=e^{-\sum_{i \in A} 1 / i}
$$

Proof. Using Lemma 3.2 we obtain an EGF of

$$
\exp \left(\log \left(\frac{1}{1-z}\right)-\sum_{i \in A} z^{i} / i\right)=\frac{1}{1-z} \prod_{i \in A} e^{-z^{i} / i}
$$

then multiplying by $(1-z)$ and taking the limit as $z \rightarrow 1$ gives the desired result.

This offers confirmation of Corollary 3.1 when substituting $A=\{k\}$. A permutation with no fixed points is called a derangement. Using a similar strategy, we can calculate the probability of a derangement.

Corollary 3.4. Let $\pi$ be a permutation taken at random from $S_{n}$. The probability that $\pi$ is a derangement is $1 / e$ in the limit as $n \rightarrow \infty$.

Proof. Just apply Corollary 3.3 to the case of cycle length 1. 
Suppose we wish to consider if a permutation has exactly $t$ cycles of length from a set $C \subset \mathbb{Z}^{+}$, in other words, all the other cycles are of length not found in $C$. In that case, we can consider such a permutation $\pi$ as a product of $\pi_{A}$ and $\pi_{B}$ such that $\pi_{A}$ has only $t$ cycles of length found in $A$, and nothing else, and $\pi_{B}$ has only cycles of length not found in $A$. This is termed by Flajolet and Sedgewick as a "labelled product"1 and and a discussion of that is found in Section II.2 in [12]. The EGF of a labelled product is merely the product of the EGFs.

Theorem 3.5. Let $\pi$ be a permutation taken at random from $S_{n}$. The probability that $\pi$ has c fixed points is $\frac{1}{c ! e}$.

Proof. Consider $\pi=\pi_{A} \pi_{B}$, where $\pi_{A}$ consists of exactly $c$ fixed points, and $\pi_{B}$ is a derangement of the remaining $n-c$ points. We must compute the labelled product $f(z)=\mathcal{P}_{e}^{(\{1\},\{c\})} \cdot \mathcal{P}_{e}^{\left(\mathbb{Z}^{+}-\{1\}, \mathbb{Z}^{\geq 0}\right)}$. Thus, by the Strong and Weak Cycle Structure Theorems,

$$
f(z)=\frac{z^{c}}{c !} \exp \left(\log \left(\frac{1}{1-z}\right)-z\right)=\frac{z^{c}}{(1-z) c !} e^{-z}
$$

An application of Thm 2.4 provides the result:

$$
\lim _{z \rightarrow 1^{-}}(1-z) f(z)=\lim _{z \rightarrow 1^{-}} \frac{z^{c}}{c !} e^{-1}=\frac{1}{c ! e}
$$

\subsection{On Cycles in Iterated Permutations}

Theorem 3.6. Let $\pi$ be a permutation in $S_{n}$. A point $x$ is a fixed point for $\pi^{k}$ if and only if $x$ is a member of a cycle of length $i$ in $\pi$, for some positive integer $i$ dividing $k$.

Proof. Write $\pi$ in disjoint cycle notation, and then $x$ appears in only one cycle (hence the name "disjoint.") Call this cycle $\psi$. Since all other cycles do not contain $x$, then $\pi^{m}(x)=\psi^{m}(x)$ for all integers $m$. Of course, $\psi$ is of order $i$ in $S_{n}$, thus $\psi^{i}=i d$, the identity element of $S_{n}$.

If $x$ is in a cycle of length $i$ then that means that $i$ is the smallest positive integer such that $\psi^{i}(x)=x$. Write $k=q i+r$ with $0 \leq r<i$. Then

$$
x=\psi^{k}(x)=\psi^{r}\left(\psi^{i q}(x)\right)=\psi^{r}\left(\left(\psi^{i}\right)^{q}(x)\right)=\psi^{r}\left(i d^{q}(x)\right)=\psi^{r}(i d(x))=\psi^{r}(x)
$$

so $\psi^{r}(x)=x$ but we said that $i$ is the least positive integer such that $\psi^{i}(x)=x$ and $r<i$. The only way this is possible is if $r$ is not positive, i.e. it is zero. Thus $k=q i$ or $i$ divides $k$.

There reverse assumes that $i$ divides $k$ so write $i q=k$ then

$$
\psi^{k}(x)=\psi^{i q}(x)=\left(\psi^{i}\right)^{q}(x)=(i d)^{q}(x)=i d(x)=x
$$

\footnotetext{
${ }^{1} \mathrm{~A}$ labelled product can be thought of as follows. If the EGF of $a(z)=b(z) c(z)$, where $b$ and $c$ are also EGFs, then $a(z)=\sum_{k=0}^{k=n}\left(\begin{array}{l}n \\ k\end{array}\right) b_{k} c_{n-k}$. Here, after building our combinatorial object in class $a$ of size $n$ out of 'an object' from $b$ of size $k$, and 'an object' from $c$ of size $n-k$, we must then attach $k$ of the $n$ labels to the former, and attach the remaining $n-k$ labels to the latter. There are precisely $\left(\begin{array}{l}n \\ k\end{array}\right)=\left(\begin{array}{c}n \\ n-k\end{array}\right)$ ways to do that.
} 
An Example Before we continue, observe what happens to a cycle of $\pi$ when evaluating $\pi^{2}$. First, if the cycle is of odd length,

$$
\left(x_{1}, x_{2}, \ldots, x_{2 c+1}\right) \mapsto\left(x_{1}, x_{3}, x_{5}, \ldots, x_{2 c+1}, x_{2}, x_{4}, x_{6}, \ldots, x_{2 c}\right)
$$

but if the cycle is of even length,

$$
\left(x_{1}, x_{2}, \ldots, x_{2 c}\right) \mapsto\left(x_{1}, x_{3}, x_{5}, \ldots, x_{2 c-1}\right)\left(x_{2}, x_{4}, x_{6}, x_{8}, \ldots, x_{2 c}\right)
$$

One can rephrase Theorem 3.6 as follows:

Corollary 3.7. Let $\pi$ be a permutation from $S_{n}$. Let $k$ be a positive integer, and let the set of positive integer divisors of $k$ be D. Then the set of fixed points of $\pi^{k}$ is precisely the set of points under $\pi$ in cycles of length found in $D$.

\subsection{Limited Cycle Counts}

Theorem 3.8. Let $k$ be a positive integer, and $\pi$ a permutation from $S_{n}$. The expected number of fixed points of $\pi^{k}$ is $\tau(k)$, taken in the limit as $n \rightarrow \infty$. Note, $\tau(k)$ is the number of positive integers dividing $k$.

Proof. We shall construct a double EGF, $a(y, z)$, where the coefficient of $y^{s} z^{t}$ is the probability that the $k^{t h}$ power of a random permutation of $S_{s+t}$ has $s$ fixed points. Let $\pi$ be a permutation taken at random from $S_{n}$. A point $x$ is a fixed point under $\pi^{k}$ if and only if $x$ is a member of a cycle of order dividing $k$ under $\pi$, via Corollary 3.6. Thus $\pi^{k}$ has exactly $t$ fixed points if and only if $\pi=\pi_{A} \pi_{B}$, where $\pi_{A} \in S_{t}$ consists only of cycles of length dividing $k$, and $\pi_{B} \in S_{n-t}$ consists only of cycles of length not dividing $k$. Let $D_{k}$ be the set of all positive divisors of $k$. The double EGF that counts the number of such permutations $\pi_{A} \pi_{B}$ will be given by the labelled product $\mathcal{P}_{e}^{\left(D_{k}, \mathbb{Z}^{\geq 0}\right)}(y) \cdot \mathcal{P}_{e}^{\left(\mathbb{Z}^{+}-D_{k}, \mathbb{Z}^{\geq 0}\right)}(z)$. By the Weak Cycle Structure Theorem and Lemma 3.2, we obtain:

$$
\begin{aligned}
a(y, z) & =\exp \left(\sum_{i \mid k} \frac{y^{i}}{i}\right) \exp \left(\log \left(\frac{1}{1-z}\right)-\sum_{i \mid k} \frac{z^{i}}{i}\right) \\
& =\exp \left(\log \left(\frac{1}{1-z}\right)\right) \exp \left(\sum_{i \mid k} \frac{y^{i}}{i}-\sum_{i \mid k} \frac{z^{i}}{i}\right) \\
& =\frac{1}{1-z} \exp \left(\sum_{i \mid k} \frac{y^{i}-z^{i}}{i}\right) .
\end{aligned}
$$

Theorem 2.5 provides the correct expected value. First observe that

$$
a_{y}(y, z)=\frac{1}{1-z} \exp \left(\sum_{i \mid k} \frac{y^{i}-z^{i}}{i}\right) \sum_{i \mid k} y^{i-1} .
$$


The plaintext is $P_{0}, \ldots, P_{31}$ and the ciphertext is $C_{0}, \ldots, C_{31}$. The internal state after round $i$ is given by $L_{0+i}, L_{1+i}, L_{2+i}, \ldots, L_{31+i}$.

$$
\begin{array}{rlrl}
L_{i}= & P_{i} & & \forall i \in[0,31] \\
& & & \forall i \in[32,559] \\
L_{i}= & k_{i-32 \bmod _{64}+L_{i-32}+L_{i-16}} & & \\
& +N L F\left(L_{i-1}, L_{i-6}, L_{i-12}, L_{i-23}, L_{i-30}\right) & \\
C_{i}= & L_{i-528} & & \forall i \in[528,559]
\end{array}
$$

where NLF stands for "non-linear function", and is given by

$N L F(a, b, c, d, e)=d+e+a c+a e+b c+b e+c d+d e+a d e+a c e+a b d+a b c$

Figure 1. The Specification of Keeloq

Then $a_{y}(z, z)=\frac{1}{1-z} \exp (0) \sum_{i \mid k} z^{i-1}$. Finally,

$$
\lim _{z \rightarrow 1^{-}}(1-z) a_{y}(z, z)=\lim _{z \rightarrow 1^{-}} \sum_{i \mid k} z^{i-1}=\sum_{i \mid k} 1=\tau(k) .
$$

\section{Application to Keeloq}

\subsection{What is Keeloq?}

Keeloq is a block cipher, with 32-bit plaintext and ciphertext blocks and a 64-bit key. It has been used in the remote keyless entry systems of many manufacturers of automobiles, and several papers have been written about it [5] [6] [7] [8] [10] [11] [3, Ch. 2]. [9]. It has 528 rounds, which is unusually high, and this can be written $528=8 \times 64+16$, a decomposition whose utility will be apparent shortly. Each round is like a stream cipher, in the sense that the internal state is a 32-bit register, and is shifted one bit, and a new bit is introduced. The new bit is a function of certain bits of the internal state, and a single bit of the key, via a map described by a cubic polynomial over GF(2), see for example [2] [3, Ch. 2]. The initial value of the internal state is the plaintext, and the final value is the ciphertext. For completeness, the cipher specification is given in Figure 1.

Also, because each round only uses 1 bit of the key (and they are used in sequence), then after 64 rounds, the entire key has been used. Therefore, it makes sense to define $f_{k}$, a function which represents those 64 rounds. Each additional 64 rounds behaves identically. It turns out that $f_{k}$ is a permutation. The remaining 16 rounds are written as $g_{k}$, which is also a permutation. Of course if either $f_{k}$ or $g_{k}$ were not permutations, then the block cipher would not be uniquely decodable. 
Thus we can write $g_{k}\left(f_{k}^{(8)}(p)\right)=E_{k}(p)$ and this motivated the authors' initial interest in iterated permutations. Also it is noteworthy that only 16 bits of the key are used by $g_{k}$, thus only 16 bits of the key need be known or guessed to use $g_{k}^{-1}$ to "peel off" or "undo" these 16 rounds, leaving us with $f_{k}^{(8)}$, the eighth iterate of a permutation.

\subsection{Bard's Dissertation Attack}

This attack assumes some portion of the code-book is available. So long as two fixed points are found, the attack can succeed. One can show that if there are two plaintexts that are fixed on the first 64 rounds of the encryption, i.e. $f\left(p_{1}\right)=p_{1}$ and $f\left(p_{2}\right)=p_{2}$, then this is sufficient information to perform an algebraic cryptanalysis, see [3, Ch. 2] [2, Ch. 3]. One writes polynomials for those two equalities and uses SAT-solvers to solve them, see [4] [2, Ch. 6].

The question becomes how to obtain those pairs. First, the part of the key used in $g_{k}$, which is 16 bits in length, is simply guessed. This has success probability $2^{-16}$. Then $g_{k}^{-1}$ can be used. This allows for $(p, c)$, the plaintext-ciphertext pairs in the codebook to be replaced by $\left(p, g_{k}^{-1}(c)\right)$ which are now actually $\left(p, f_{k}^{(8)}(p)\right)$. These are points fixed by $f_{k}^{(8)}$ and so by Corollary 3.6, they are points of order $\{1,2,4,8\}$ for $f_{k}$. Thus, the fixed points of $f_{k}$, which are useable for the cryptanalysis, are a subset of those for $f_{k}^{(8)}$, which we can find.

Theorem 4.1. Let $\pi$ be a random permutation from $S_{n}$. The probability that $\pi$ has $c_{1}$ fixed points and $c_{2}$ cycles of lengths 2,4 , or 8 , is given by

$$
\frac{1}{c_{1} ! c_{2} !}\left(\frac{7}{8}\right)^{c_{2}} e^{-15 / 8}
$$

Proof. Note that the set of permutations on $n$ elements, with $c_{1}$ fixed points, and $c_{2}$ cycles of length 2,4 , or 8 , can be thought of as a triple labelled product. The first item in the product is from $\mathcal{P}^{\left(\{1\}, c_{1}\right)}$, the second item from $\mathcal{P}^{\left(\{2,4,8\}, c_{2}\right)}$, and the third item from $\overline{\mathcal{P}} \overline{\{1,2,4,8\}}$. We must now calculate the EGF.

The first item has $\alpha(z)=z$, and $\beta(z)=z^{c_{1}} / c_{1}$ !, for an EGF of $\beta(\alpha(z))=z^{c_{1}} / c_{1}$ ! The second item has $\alpha(z)=z^{2} / 2+z^{4} / 4+z^{8} / 8$, and $\beta(z)=z^{c_{2}} / c_{2}$ !, therefore an EGF of $\beta(\alpha(z))=\frac{1}{c_{2} !}\left[z^{2} / 2+z^{4} / 4+z^{8} / 8\right]^{c_{2}}$. Finally, the third item has EGF given by Lemma 3.2,

$$
\exp \left(\log \left(\frac{1}{1-z}-\sum_{i \mid 8} z^{i} / i\right)\right)=\frac{1}{1-z} \exp \left(-\sum_{i \mid 8} z^{i} / i\right)
$$

giving a final, total EGF of

$$
\frac{z^{c_{1}}}{(1-z) c_{1} ! c_{2} !}\left[\frac{z^{2}}{2}+\frac{z^{4}}{4}+\frac{z^{8}}{8}\right]^{c_{2}} \exp \left(-\sum_{i \mid 8} z^{i} / i\right)
$$




$\begin{array}{cccccc}\eta & 10 \% & 20 \% & 30 \% & 40 \% & 50 \% \\ \text { Success } & 0.47 \% & 1.75 \% & 3.69 \% & 6.16 \% & 9.02 \% \\ & & & & & \\ \eta & 60 \% & 70 \% & 80 \% & 90 \% & 100 \% \\ \text { Success } & 12.19 \% & 15.58 \% & 19.12 \% & 22.75 \% & 26.42 \%\end{array}$

Table 1. Success Probabilities of Bard's Dissertation Attack

Multiplying by $1-z$ and taking the limit as $z \rightarrow 1^{-}$, via Theorem 2.4 we obtain

$$
\frac{1}{c_{1} ! c_{2} !}\left[\frac{1}{2}+\frac{1}{4}+\frac{1}{8}\right]^{c_{2}} \exp (-\sigma(8) / 8)=\frac{1}{c_{1} ! c_{2} !}(7 / 8)^{c_{2}} e^{-15 / 8}
$$

The method requires $c_{1} \geq 2$, otherwise the attack fails. This can be easily calculated as $1-\operatorname{Pr}\left\{c_{1}=0\right\}-\operatorname{Pr}\left\{c_{1}=1\right\} \approx 0.2642$ probability of success.

Second, suppose that $\eta$ is the fraction of the code-book available. Then any given fixed point is found with probability $\eta$ in the known part of the code-book, and so at least two will be found with probability

$$
1-\left(\begin{array}{c}
c_{1} \\
0
\end{array}\right) \eta^{0}(1-\eta)^{c_{1}}-\left(\begin{array}{c}
c_{1} \\
1
\end{array}\right) \eta^{1}(1-\eta)^{c_{1}-1}=1-(1-\eta)^{c_{1}-1}\left[1-\left(c_{1}+1\right) \eta\right]
$$

and so the following $\eta$ and success probabilities can be found, generated by Theorem 3.5 and listed in Table 4.2. Note, these are absolute probabilities, not probabilities given $c_{1} \geq 2$.

Using Maple, one can also calculate exactly when the probability of having the two fixed points in the $\eta$ fraction of the code-book is one-half. This is at $\eta=63.2 \%$ remarkably close to the empirical calculation in [3, Ch. 2].

Note that while finding two fixed points of $f_{k}$ is enough to break the cipher, using SAT-solvers as noted above, the fixed points of $f_{k}^{(8)}$ are still an annoyance. Our postprocessed code-book will have all the fixed points of $f_{k}^{(8)}$ in it, and at worst we must try all pairs.

If $\pi$ has $c_{1}$ fixed points, and $c_{2}$ cycles of length 2,4 , or 8 , then $\pi^{8}$ has at most $c_{1}+8 c_{2}$ fixed points, as each cycle of length 2 produces 2 , of length 4 produces 4 , and of length 8 produces 8 . Thus of the $c_{2}$ cycles of length 2 , or 4 , or 8 , at most $8 c_{2}$ fixed points are produced. This means in the code-book we have at most $c_{1}+8 c_{2}$ fixed points, or $\left(c_{1}+8 c_{2}\right)\left(c_{1}+8 c_{2}-1\right) / 2$ pairs of them. At absolute worst, we have to check all of them. The expected value of the number of pairs, given $c_{1} \geq 2$ can be calculated with Maple, and is $113 / 2-105 / e \approx 17.87$. As each pair takes less than a minute, this is not the rate-determining step.

The post-processing of the code-book will take much more time, $\eta 2^{32}$ Keeloq encryptions, but this is still much smaller than brute-forcing the $2^{64}$ keys. 


\subsection{The Courtois-Bard-Wagner Attack}

Again, in this attack (first published in [11]), we will iterate over some portion of the code-book. One property of the cipher Keeloq, is that only one bit is changed per round. Thus the last sixteen rounds, represented by $g_{k}(x)$, only affect sixteen bits of the ciphertext. Thus, if $x$ is a fixed point of $f_{k}^{(8)}$, then 48 out of the 64 bits will match, compared between the plaintext and the ciphertext. One can easily scan for this property.

This matching property will always occur for a fixed point of $f_{k}^{(8)}$, but it happens by coincidence with probability $2^{-16}$. Therefore, the number of code-book entries with this property will be the number of fixed points of $f_{k}^{(8)}$, plus an expected $2^{-16} 2^{32}=2^{16}$ "red herrings". What is remarkable, is that [11] contains a formula for the 16 key bits that would cause the effect if it were because the plaintext were a fixed point (i.e. not a coincidence). Therefore, each code-book entry with the matching property can be tagged with a 16-bit potential sub-key.

As it turns out, the 16-sub key, as well as any single plaintext-ciphertext pair that is a fixed point of $f_{k}$, not merely of $f_{k}^{(8)}$, is enough to mount an algebraic attack. Thus we have the following steps. Let $c_{3}$ denote the number of fixed points of $f^{(8)}$.

(1) Check all $2^{32}$ code-book entries for the matching property.

(2) Of these (roughly $2^{16}+c_{3}$ ) plaintext-ciphertext pairs, compute the sub-key that they imply.

(3) For each plaintext-ciphertext pair with the property, set up an algebraic cryptanalysis problem with the one pair, assuming it is a fixed point of $f$, and assuming the sub-key is correct.

(4) If an answer is obtained, verify assumptions. If assumptions turned out to be false, or if the problem is "unsatisfiable", go to Step 3.

Sorting upon this sub-key between Step 2 and Step 3 would reveal which are the

likely pairs, as the same sub-key will tag all the fixed points of $f_{k}$ and $f_{k}^{(8)}$. We expect each of the $2^{16}$ "red-herrings" to be tagged with uniformly randomly distributed potential sub-keys. Therefore, in the first very few Step 3 and Step 4 executions, we would obtain the key.

What is needed for success? First, that $f_{k}$ have at least one genuine fixed point. This occurs with probability $1-1 / e$, as proven in Corollary 3.4 , and is roughly 0.6321 . Second, the expected amount of work in Step 1 is at most $2^{32}$ Keeloq Encryptions, and a more precise estimate is found in [11]. Third, Step 2 is negligible. Fourth, for Step 3 and Step 4, we must execute these stages for each potential sub-key. Given the model of the previous attack, and using Theorem 4.1, we can obtain a bound on the expected number of repetitions of Steps 3 and 4 . This is upper-bounded by the expected value of $c_{1}+8 c_{2}$ given that $c_{1}>0$. Using Maple, this comes to $113 / 2-46 / e \approx 39.58$, the difference being that we now allow $c_{1}=1$, which was previously forbidden. Of course, without the sorting explained in the previous paragraph, the expected number of Step 3 and Step 4 executions would be around $2^{15}$. 


\section{Highly Iterated Ciphers}

Here we present two attacks, which while no where near practical feasibility, present surprising results that the authors did not anticipate.

Suppose there were three naïve cryptography students, who choose to use 3-DES iterated $^{2}$ approximately one million times, because they are told that this will slow down a brute force attacker by a factor of one million. Alice will choose $1,000,000$ iterations, Bob will choose 1,081,079 iterations and Charlie will choose 1,081,080 iterations. Intuitively, one would not expect these three choices to have significantly different security consequences.

However, assuming that the 3-DES cipher for a random key behaves like a randomly chosen permutation from $S_{2^{64}}$, these permutations will have

$$
\tau(1,000,000)=49 \quad \tau(1,081,079)=2 \quad \tau(1,081,080)=256
$$

fixed points which allows for the following distinguisher attack. It is noteworthy that Charlie's number is the lowest positive integer $x$ to have $\tau(x)=256$, while Bob's number (only one less) is prime, and thus has $\tau(x-1)=2$. This enables the dramatic difference in vulnerability to the attack.

In a distinguishing attack, the attacker is presented either with a cipher, or with a random permutation from the set of those with the correct domain. Randomly iterate through 1/64 of the plain-space. If a fixed point is found, guess that one is being given a user cipher. If no fixed point is found, guess random.

In the case of Alice's implementation, there will be an expected value of $\approx 0.766$ fixed points. In the case of Bob's, $1 / 32$ expected fixed points. In the case of Charlie's, 4 expected fixed points. A random permutation would have 1/64 expected fixed points. Thus, we can see that Charlie's would be easily distinguishable from a random permutation, but Bob's much less so. Against Alice, the attack could definitely still be mounted but with an intermediate probability of success. To make this notion precise, we require the probability distribution of the number of fixed points of $\pi^{k}$. In fact, one can prove the following

Theorem 5.1. Let $\pi \in S_{n}$ be a permutation chosen at random, then the $c^{\text {th }}$ term of the following EGF

$$
\exp \left(\sum_{i \mid k} \frac{y^{i}-1}{i}\right)
$$

is the probability that $\pi^{k}$ has exactly c fixed points.

Proof. Consider the double EGF of Theorem 3.8, $a(y, z)=\frac{1}{1-z} \exp \left(\sum_{i \mid k} \frac{y^{i}-z^{i}}{i}\right)$. Recall, the coefficient of $y^{s} z^{t}$ is the probability that $\pi^{k} \in S_{s+t}$ has $s$ fixed points. Now, for any given $s$, we can find the probability that $\pi^{k} \in S_{n}$ has $s$ fixed points (in the limit as $n \rightarrow \infty)$, by evaluating $\lim _{z \rightarrow 1^{-}}(1-z) a(y, z)$. The result is the EGF $\exp \left(\sum_{i \mid k} \frac{y^{i}-1}{i}\right)$.

\footnotetext{
${ }^{2}$ Since the brute force attack is the optimal attack known at this time, it is perhaps not completely unreasonable. The classic UNIX implementations encrypt with a variant of DES 25 times, for example [13, Ch. 8].
} 
However, the above requires us to have 256 terms inside of the exponentiation, for there are 256 positive integers dividing 1,081,080, and we will need to know the coefficient of the $c^{\text {th }}$ term for at least 1000 terms. Therefore, we are compelled to leave this as a challenge for the computer algebra community.

Meanwhile, we performed the following experiment. We generated 10,000 random permutations $\pi$ from $S_{10,000}$ and raised $\pi$ to the $k$ th power for the values of $k$ listed. Then we calculated $c$, the number of fixed points of $\pi^{k}$, and determined if a search of the first $1 / 64$ th of the domain would reveal no fixed points. That probability is given by

$$
(1-c / n)^{n / 64} \approx e^{-c / 64}
$$

and taking the arithmetic mean over all experiments, one obtains

$\begin{array}{rlll} & \text { No fixed points } & \text { One or more } & \\ k=1 & 0.985041 & 0.014959 & \text { Random } \\ k=1000000 & 0.797284 & 0.202716 & \text { Alice } \\ k=1081079 & 0.984409 & 0.015591 & \text { Bob } \\ k=1081080 & 0.418335 & 0.581665 & \text { Charlie }\end{array}$

Perhaps this is unsurprising, as in the case of Charlie, we expect 256 fixed points, and so it would be surprising if all of those were missing from a part of the domain equal to 1/64th of the total domain in size. On the other hand, for Bob we expect only 2 fixed points, and it is exceptional that we find one by accident.

Finally, we observe that if there is an equal probability of an adversary being presented with a random cipher from $S_{2^{64}}$ or 3-DES in the key of one of our three users, iterated to their exponent, then the success probability of the attacker would be for Alice $59.39 \%$, for Bob 50.03\%, and for Charlie $78.34 \%$. Note in each case, we check only $2^{64} / 64=2^{58}$ plaintexts, and so this attack is $2^{112} / 2^{58}=2^{54}$ times faster than brute-force.

A General Maxim: If a permutation must be iterated for some reason, then it should be iterated a prime number of times, to avoid fixed points.

\subsection{A Key Recovery Attack}

Consider the cipher given by

$$
F_{k_{1}, k_{2}}(p)=E_{k_{1}}\left(E_{k_{2}}^{(n)}\left(E_{k_{1}}(p)\right)\right)=c
$$

where $k_{1}$ and $k_{2}$ are keys, and $E$ is encryption with a block cipher (let $D_{k}(c)=p$ denote decryption). If $E$ is DES and $n=1$, then this is the "triple DES" construction. Here, we consider that $E$ is AES-256 as an example, and $n$ is Charlie's number, 1081080. Then $F$ is a block cipher with 512-bit key and 128-bit plaintext block. We will refer to $k_{1}$ as the outer key, and $k_{2}$ as the inner key.

Suppose an attacker had an oracle for $F$ that correctly encrypts with the correct $k_{1}$ and $k_{2}$ that the target is using. Call this oracle $\phi(p)$. Observe that $G_{k_{3}}(x)=$ 
$D_{k_{3}}\left(\phi\left(D_{k_{3}}(x)\right)\right)$ will have $G_{k_{3}}(x)=E_{k_{2}}^{(n)}(x)$ if and only if $k_{3}=k_{1}$. Thus if we can correctly guess the outer key, we have an oracle for the $n$th iteration of encryption under the inner key. If $k_{3} \neq k_{1}$, then provided that $E_{k_{1}}$ is computationally indistinguishable from a random permutation from $S_{2^{128}}$ when $k_{1}$ is chosen uniformly at random (a standard assumption) then $G_{k_{3}}(x)$ also behaves as a random permutation.

Thus, for $k_{1}=k_{3}$, we can expect $G_{k_{3}}(x)$ to behave like Charlie's cipher in the previous section, and for $k_{1} \neq k_{3}$, we can expect $G_{k_{3}}(x)$ to behave like a random permutation in the previous section.

Let one run of the distinguishing attack signify guessing all possible $k_{3}$ values, and executing the previous section's attack for each key. If "random" is indicated (i.e. no fixed point found), then we reject the $k_{3}$ but if "real" is indicated (i.e. at least one fixed point found), then we add $k_{3}$ to a "candidate list."

After one run of this distinguishing attack, we would have a candidate list of outer keys of expected size

$$
(0.014959)\left(2^{256}-1\right)+(0.581665)(1)
$$

where the success probabilities are given in the previous section, for the attack on Charlie.

If we repeat the distinguisher attack on these candidate keys, taking care to use a distinct set of plaintexts in our search, the success probabilities will be the same. This non-overlapping property of the plaintext search could be enforced by selecting the six highest-order bits of the plaintext to be the value of $n$. After $n$ runs, we would expect the list to contain

$$
(0.014959)^{n}\left(2^{256}-1\right)+(0.581665)^{n}(1)
$$

candidate keys.

Of course, the true $k_{3}=k_{1}$ key will be present with probability $0.581665^{n}$. Next, for each key $k_{c}$ on the candidate list, we will check all possible $2^{256}$ values of $k_{2}$ (denoted $k_{x}$ ), via checking if

$$
p=\phi\left(D_{k_{c}}\left(D_{k_{x}}^{(n)}\left(D_{k_{c}}(p)\right)\right)\right)
$$

which will be true if $k_{x}=k_{2}$ and $k_{c}=k_{1}$. This check should be made for roughly 4-6 plaintexts, to ensure that the match is not a coincidence. This necessity arises from the fact that the cipher has a 512-bit key and 128-bit plaintext. We will be very conservative, and select 6 .

The number of encryptions required for the $n$ runs is

$$
\begin{array}{r}
(1081080+4)\left(\frac{2^{128}}{64}\right)\left(2^{256}+(0.014959)\left(2^{256}\right)+(0.014959)^{2}\left(2^{256}\right)+\right. \\
\left.(0.014959)^{3}\left(2^{256}\right)+\cdots+(0.014959)^{n}\left(2^{256}\right)\right) \\
=(1081080+2)\left(2^{378}\right) \frac{1-(0.014959)^{n+1}}{1-0.014959} \\
=2^{398.06579 \cdots}\left(1-0.014959^{n+1}\right)
\end{array}
$$


and for the second stage

$$
\begin{array}{r}
(6)(2)(2+1081080)\left(2^{256}\right)\left(0.014959^{n}\right)\left(2^{256}\right)=\left(2^{535.6290 \cdots}\right)\left(0.014959^{n}\right) \\
=2^{535.6290-6.062842 n}
\end{array}
$$

for a success probability of $(0.581665)^{n}$.

Using Maple, we find that $n=23$ is optimal, leaving a candidate list of $2^{116.555 \cdots}$ possible keys, and requiring $2^{398.41207 \cdots}$ encryptions, but with success probability $(0.581665)^{23} \approx 2^{-17.98001 \cdots}$. A brute-force search of the $2^{512}$ possible keys would have $(6)(2)(1081082) 2^{512}$ encryptions to perform, or $2^{535.629007 \ldots}$. Naturally, if a success probability of $2^{-17.98001 \cdots}$ were desired, then only $2^{517.649 \cdots}$ encryptions would be needed for that brute-force search.

Therefore this attack is $2^{119.237}$ times faster than brute-force search.

\section{Conclusions}

In this paper, we presented a known theorem on the probabilities of random permutations having given cycle structures and cycle counts, along with several useful corollaries. To demonstrate the applicability of this technique to cryptanalysis, we have taken two attacks which were heretofore presented at least partially heuristically, and made them fully rigorous. It is hoped that other attacks which rely upon detecting these probabilities via experimentation will be made rigorous as well, by calculation via EGFs and OGFs. We also hope that we have demonstrated the utility of analytic combinatorics in general, as well as EGFs and OGFs in particular.

We also presented a new attack, on very highly iterated permutations. While the scenario is not reasonable, and it is only a distinguisher attack, it is also interesting that the $\tau$ function occurs here. If a permutation should be highly iterated, it should be iterated a prime number of times. However, the choice of 25 on the part of UNIX designers was not bad, as $\tau(25)=3$. We also extended this to a key-recovery attack, in an unusual context. It is unclear in what situations such large numbers of iterations would occur, but from a pure mathematical point of view, the additional security granted by prime iteration counts is interesting.

Acknowledgments. We thank Sebastiaan Indesteege, a graduate student from Katholieke Universiteit Leuven in Belgium, for helpful comments; Sean O'Neil, an independent scientist from Ireland, also for helpful comments; Dr. Kenneth Patterson of the Royal Holloway University of London for questions at the 2008 Workshop on Mathematical Cryptography in Santander, Spain, that encouraged us to rigorously describe the attack in Section 5; and Prof. Philippe Flajolet and Prof. Robert Sedgewick, for their excellent text on analytic combinatorics [12].

\section{References}

[1] Apéry, R. "Irrationalité de $\zeta(2)$ et $\zeta(3) . "$ Astérisque 61, 11-13, 1979. 
[2] Gregory V. Bard. Algebraic Cryptanalysis. Springer-Verlag. (Scheduled Release) 2009.

[3] Gregory V. Bard. Algorithms for the Solution of Linear and Polynomial Systems of Equations over Finite Fields, with Applications to Cryptanalysis. PhD Dissertation. Department of Applied Mathematics and Scientific Computation, University of Maryland at College Park. Defended April 30, 2007.

[4] Gregory V. Bard, Nicolas T. Courtois and Chris Jefferson: Efficient Methods for Conversion and Solution of Sparse Systems of Low-Degree Multivariate Polynomials over GF(2) via SATSolvers, Available at http://eprint.iacr.org/2007/024/.

[5] Eli Biham, Orr Dunkelman, Sebastiaan Indesteege, Nathan Keller, Bart Preneel: How to Steal Cars - A Practical Attack on KeeLoq, in Eurocrypt 2008, LNCS 4965, pp. 1-18, Springer, 2008.

[6] Andrey Bogdanov: Cryptanalysis of the KeeLoq block cipher, http://eprint.iacr.org/2007/055.

[7] Andrey Bogdanov: Attacks on the KeeLoq Block Cipher and Authentication Systems, 3rd Conference on RFID Security 2007, RFIDSec 2007.

[8] Andrey Bogdanov: Linear Slide Attacks on the KeeLoq Block Cipher, The 3rd SKLOIS Conference on Information Security and Cryptology (Inscrypt 2007), LNCS, Springer-Verlag, 2007

[9] Nicolas Courtois, Gregory V. Bard and Andrey Bogdanov: Periodic Ciphers with Small Blocks and Cryptanalysis of KeeLoq, In Tatra Mountains Mathematic Publications, post-proceedings of Tatracrypt 2007 conference, to appear in 2008.

[10] Nicolas Courtois, Gregory V. Bard, David Wagner: Algebraic and Slide Attacks on KeeLoq, Older preprint with an incorrect specification of KeeLoq, available at eprint.iacr.org/2007/062/.

[11] Nicolas Courtois, Gregory V. Bard, David Wagner: Algebraic and Slide Attacks on KeeLoq, In FSE 2008, pp. 97-115, LNCS 5086, Springer. Older (partly out of date) preprint available at eprint.iacr.org/2007/062/.

[12] Philippe Flajolet and Robert Sedgewick; Analytic Combinatorics, book of 807 pages, to apear in Cambridge University Press in the first half of 2008. Available in full on the Internet, see http://algo.inria.fr/flajolet/Publications/book.pdf

[13] Simson Garfinkel and Gene Spafford. Practical Unix \& Internet Security. O’Reilly. 2nd edition. 1996.

[14] Maple: A Computer Algebra System. http: //www . maplesoft . com/.

[15] Marko R. Riedel, Random Permutation Statistics, paper available on the internet, at http://www.geocities.com/markoriedelde/papers/randperms.pdf.

[16] Weisstein, Eric W. "Apéry's Constant." From MathWorld-A Wolfram Web Resource. http://mathworld.wolfram.com/AperysConstant.html

\section{A Of Pure Mathematical Interest}

The authors encountered the following interesting connections with some concepts in number theory, but they turned out to be not needed in the body of the paper. We present them here for purely scholarly interest. 


\section{A.1 The Sigma Divisor Function}

Lemma A.1. The sum $\sum_{i \mid k} 1 / i=\frac{1}{k} \sigma(k)$ where both $i$ and $k$ are positive integers, and where $\sigma(k)$ is the divisor function (i.e. the sum of the positive integers which divide $k$ ).

Proof.

$$
\sum_{i \mid k} 1 / i=\frac{k}{k} \sum_{i \mid k} 1 / i=\frac{1}{k} \sum_{i \mid k} k / i=\frac{1}{k} \sum_{i \mid k} i=\frac{1}{k} \sigma(k)
$$

Corollary A.2. Let $\pi$ be a permutation taken at random from $S_{n}$. The probability that $\pi^{k}$ is a derangement is $e^{-\sigma(k) / k}$, in the limit as $n \rightarrow \infty$.

Proof. Let $D$ be the set of positive integers dividing $k$. From Corollary 3.7, we know that $x$ is a fixed point of $\pi^{k}$ if and only if $x$ is in a cycle of length found in $D$ for $\pi$.

We will use Corollary 3.2, with $\bar{A}=D$. We obtain the probability is $e^{-\sum_{i \in D} 1 / i}$, and Lemma A.1 gives the desired result.

Note that substituting $A=\{1\}$ into the above yields the same result as Corollary 3.4.

\section{A.2 Apéry's Constant}

Corollary 3.3 provides an amusing connection with Riemann's zeta function. Recall, for complex $s$, the infinite series, $\sum_{n \geq 1} 1 / n^{s}$ defines the "zeta function" $\zeta(s)$, provided the series converges.

Corollary A.3. The probability that a random permutation (in the limit as the size grows to infinity) does not contain cycles of square length is:

$$
e^{-\sum_{i \geq 1} 1 / i^{2}}=e^{-\zeta(2)}=e^{-\pi^{2} / 6} \approx 0.19302529,
$$

or roughly $1 / 5$.

Corollary A.4. The probability that a random permutation (in the limit as the size grows to infinity) does not contain cycles of cube length is: $e^{-\zeta(3)} \approx 0.30057532$

Note, $\zeta(3)$ is known as Apéry's Constant [1], and occurs in certain quantum electrodynamical calculations, but is better known to mathematicians as being the probability that any three integers chosen at random will have no common factor dividing them all [16].

Received $\mathrm{xxx}$; revised $\mathrm{xxx}$

\section{Author information}

Nicolas T. Courtois, University College of London, Gower Street, London, WC1E6BT, UK.

Email: n.courtois@ucl.ac.uk

Gregory V. Bard, Fordham University, Department of Mathematics, The Bronx, NY, 10458, USA.

Email: bardefordham.edu

Shaun V. Ault, Fordham University, Department of Mathematics, The Bronx, NY, 10458, USA.

Email: aultefordham.edu 